\title{
77. Integrations on the Circle of Convergence and the Divergence of Interpolations. I
}

\author{
By Tetsujiro KAKEHASHI
}

(Comm. by K. KunUgi, M.J.A., June 13, 1955)

Let the points

$$
\left\{\begin{array}{l}
z_{1}^{(1)} \\
z_{1}^{(2)}, z_{2}^{(2)} \\
z_{1}^{(3)}, z_{2}^{(3)}, z_{3}^{(3)} \\
\cdots \cdots \cdots \\
z_{1}^{(n)}, z_{2}^{(n)}, z_{3}^{(n)}, \ldots, z_{n}^{(n)} \\
\ldots \ldots \ldots
\end{array}\right.
$$

which do not lie exterior to the unit circle $C:|z|=1$, satisfy the condition that the sequence of

$$
\frac{w_{n}(z)}{z^{n}}=\left(z-z_{1}^{(n)}\right)\left(z-z_{2}^{(n)}\right) \cdots\left(z-z_{n}^{(n)}\right) / z^{n}
$$

converges to a function $\lambda(z)$ single valued, analytic, and non-vanishing for $z$ exterior to $C$, and uniformly for any finite closed set exterior to $C$, that is

$$
\lim _{n \rightarrow \infty} \frac{w_{n}(z)}{z^{n}}=\lambda(z) \neq 0 \text { for }|z|>1
$$

Let $f(z)$ be a function single valued and analytic within the circle $C_{\mathrm{p}}:|z|=\rho>1$ but not analytic on $C_{\mathrm{p}}$. Then the sequence of polynomials $P_{n}(z ; f)$ of respective degrees $n$ which interpolate to $f(z)$ in all the zeros of $w_{n+1}(z)$ is known to be

$$
\text { ( I ) } \quad P_{n}(z ; f)=\frac{1}{2 \pi i} \int_{c_{R}} \frac{w_{n+1}(t)-w_{n+1}(z)}{w_{n+1}(t)} \frac{f(t)}{t-z} d t, \quad(1<R<\rho) \text {. }
$$

It is known that the sequence of polynomials $P_{n}(z ; f)$ converges to $f(z)$ throughout the interior of the circle $C_{\rho}$, and uniformly for any closed set interior to $C_{p}$. But the divergence of $P_{n}(z ; f)$ at every point exterior to $C_{\rho}$ is not established in general.

This problem is seen in the paper by Walsh: The divergence of sequences of polynomials interpolating in roots of unity; Bulletin of the American Mathematical Society, 1936, Vol. 42, p. 715. And that is treated in the following papers by the author.

T. Kakehashi: On the convergence-region of interpolation polynomials; Journal of the Mathematical Society of Japan, 1955, Vol. 7, p. 32. T. Kakehashi: The divergence of interpolations. I, II, III; Proceedings of the Japan Academy, 1954, Vol. 30, Nos. 8, 9, and 10.

In this paper, we consider a certain type of integrations on the convergence-circle of a function, which belongs to a certain class of 
functions, and consider the divergence properties of $P_{n}(z ; f)$ at every point exterior to the convergence-circle.

1. Let $F(\theta) ; 0 \leqq \theta \leqq 2 \pi$ be a complex valued function with the bounded variation (not necessarily periodic). Then the function

$$
f(z)=\frac{1}{2 \pi} \int_{0}^{3 \pi} \frac{\rho e^{i \theta}}{\rho e^{i \theta}-z} d F(\theta), \quad|z|<\rho
$$

is single valued and analytic within the circle $C_{\mathrm{p}}:|z|=\rho$.

Definition 1. Let $K_{p}(\rho>0)$ be denoted by the class of functions $f(z)$ which satisfy the conditions

$$
\begin{gathered}
f(z)=\sum_{n=0}^{\infty} c_{n}\left(\frac{z}{\rho}\right)^{n}=\frac{1}{2 \pi} \int_{0}^{3 \pi} \frac{\rho e^{i \theta}}{\rho e^{i \theta}-z} d F(\theta) \quad(\rho>0), \\
c_{n}=\frac{1}{2 \pi} \int_{0}^{2 \pi} e^{-i n \theta} d F(\theta) ; \quad n=0,1,2, \ldots,
\end{gathered}
$$

and

$$
\varlimsup_{n \rightarrow \infty}\left|c_{n}\right|>0
$$

where $F(\theta)$ is a complex valued function with the bounded variation and is normalized by

$$
F(0)=0, \quad F(\theta-0)=F(\theta) .
$$

It is clear that a function which belongs to $K_{\mathrm{p}}$ is single valued and analytic within the circle $C_{\rho}:|z|=\rho$ but not analytic on $C_{\rho}$, and that, in the power series $\sum c_{n}\left(\frac{z}{\rho}\right)^{n}$, the coefficients $c_{n}$ satisfy $0<\lim _{n \rightarrow \infty}\left|c_{n}\right|<\infty$. And the function $F(\theta)$ in (1.2) can not be absolutely continuous by the Riemann-Lebesgue theorem. It is to be noticed that the Fourier-Stieltjes coefficients $c_{-n}$ with negative suffixes of $F(\theta)$ is not considered.

For example, if $F(\theta)$ is a step function, $f(z)$ is a function with poles of first order on the circle $C_{\rho}$.

Let $f(z)$ be the function which belongs to the class $K_{r}$, defined by (1.1) and $\varphi(z)$ be a function single valued and analytic on $C_{\rho}$, and be defined by the Laurent's series

$$
\varphi(z)=\sum_{n=-\infty}^{\infty} \alpha_{n} z^{n}
$$

We can not define in general the integral

$$
\int_{C_{\mathrm{p}}} \varphi(t) f(t) d t
$$

in ordinary sense. In this case, we define the finite part of the above integral by

$$
\frac{p f .}{2 \pi i} \int_{c_{\varphi}} \varphi(t) f(t) d t=\frac{1}{2 \pi} \int_{0}^{2 \pi} \varphi^{*}\left(\rho e^{i \theta}\right) \rho e^{i \theta} d F(\theta),
$$

where $\varphi^{*}(z)$ is the principal part of the Laurent's series (1.5), that is

$$
\varphi^{*}(z)=\sum_{n=1}^{\infty} \alpha_{-n} z^{-n} \text {. }
$$


Lemma 1.1. Let $f(z)$ be a function which belongs to the class $K_{\rho}$, and $\varphi(z)$ be a function single valued and analytic on and between the two circles $C_{\mathrm{p}}:|z|=\rho$ and $C_{R}:|z|=R<\rho$. Then

$$
p f . \int_{C_{\rho}} \varphi(t) f(t) d t=\int_{G_{R}} \varphi(t) f(t) d t .
$$

By Cauchy's theorem, we have

$$
\frac{1}{2 \pi i} \int_{C_{R}} \varphi(t) f(t) d t=\frac{1}{2 \pi i} \int_{c_{R}} \varphi^{*}(t) f(t) d t=\sum_{n=0}^{\infty} c_{n} \alpha_{-n-1} \rho^{-n},
$$

where $\varphi^{*}(t), c_{n}$, and $\alpha_{-n-1}$ are defined respectively by (1.7), (1.1), and (1.7). It is clear that the last side is convergent. The left-side of (1.8) is also

$$
\frac{p f .}{2 \pi i} \int_{c_{\rho}} \varphi(t) f(t) d t=\frac{1}{2 \pi} \int_{0}^{2 \pi} \sum_{n=0}^{\infty} \alpha_{-n-1} \rho^{-n} e^{-n i \theta} d F(\theta)=\sum_{n=0}^{\infty} c_{n} \alpha_{-n-1} \rho^{-n} .
$$

Thus the lemma is established.

Lemma 1.2. Let $f(z)$ be a function which belongs to the class $K_{\mathrm{p}}$, and a function $\varphi(z)$ single valued and analytic on $C_{\mathrm{p}}$ be non-vanishing on $C_{\rho}$. Then

$$
\overline{\lim }_{n \rightarrow \infty}\left|\frac{\rho^{n} p f .}{2 \pi i} \int_{C_{\rho}} \varphi(t) f(t) t^{-n-1} d t\right|>0 .
$$

The function $1 / \varphi(z)$ which is single valued and analytic on $C_{\rho}$ can be expanded into the Laurent's series

$$
1 / \varphi(z)=\sum_{n=-\infty}^{\infty} \beta_{n}\left(\frac{z}{\rho}\right)^{n}=\sum_{n=-\infty}^{\infty} \beta_{n} e^{n i \theta},
$$

which is absolutely convergent on $C_{\rho}$. The function $\varphi(z) f(z)$ is also expanded into the Laurent's series

$$
\varphi(z) f(z)=\sum_{-\infty}^{\infty} \gamma_{n}\left(\frac{z}{\rho}\right)^{n}
$$

Then the function $f(z)$ can be expanded into

$$
f(z)=\sum_{n=0}^{\infty} c_{n}\left(\frac{z}{\rho}\right)^{n}=\sum_{n=0}^{\infty}\left(\sum_{p=-\infty}^{\infty} \gamma_{p} \beta_{n-p}\right)\left(\frac{z}{\rho}\right)^{n} .
$$

If we assume

$$
\lim _{n \rightarrow \infty} \frac{\rho^{n} p f \cdot}{2 \pi i} \int_{c_{p}} \varphi(t) f(t) t^{-n-1} d t=0,
$$

we have $\lim _{n \rightarrow \infty} \gamma_{n}=0$ by lemma 1.1. Let $M=\max \left|\gamma_{n}\right|$, then as $n$ tends to infinity

$$
\begin{gathered}
\left|c_{n}\right|=\left|\sum_{p=-\infty}^{\infty} \gamma_{p} \beta_{n-p}\right| \leqq M \sum_{p=-\infty}^{\left[\frac{n}{2}\right]}\left|\beta_{n-p}\right|+\max _{p>\frac{n}{2}}\left|\gamma_{p}\right| \sum_{p=\left[\frac{n}{2}\right]+1}^{\infty}\left|\beta_{n-p}\right| \\
\leqq M \sum_{q=n-\left[\frac{n}{2}\right]}^{\infty}\left|\beta_{q}\right|+\max _{p>\frac{n}{2}}\left|\gamma_{p}\right| \sum_{y=-\infty}^{\infty}\left|\beta_{q}\right| \rightarrow 0
\end{gathered}
$$

by the absolutely convergence of $\sum \beta_{n} e^{n i \theta}$ and $\gamma_{n} \rightarrow 0$ as $n \rightarrow \infty$, which contradicts the condition of $f(z)$. Thus the lemma has been proved. 
Lemma 1.3. Let $f(z)$ be a function of the class $K_{\mathrm{p}}$ and the sequence of $\varphi_{n}(z): n=1,2, \ldots$ single valued and analytic on $C_{\mathrm{p}}$ tend to zero uniformly on $C_{\mathrm{p}}$ as $n \rightarrow \infty$. Then

$$
\lim _{n \rightarrow \infty} \frac{\rho^{n} p f \cdot}{2 \pi i} \int_{a_{\mathrm{p}}} \varphi_{n}(t) f(t) t^{-n-1} d t=0 .
$$

Let $\psi_{n}(t) ; n=1,2, \ldots$ be the principal parts respectively of $\varphi_{n}(t) t^{-n-1}$. It is clear that $\psi_{n}(t) \rho^{n}$ tends to zero on $C_{\rho}$ as $n \rightarrow \infty$ by the condition of $\varphi_{n}(t)$.

Hence

$$
\frac{\rho^{n} p f .}{2 \pi i} \int_{c_{\mathrm{p}}} \varphi_{n}(t) f(t) t^{-n-1} d t=\frac{1}{2 \pi} \int_{0}^{2 \pi} \psi_{n}\left(\rho e^{i \theta}\right) \rho e^{i \theta} d F(\theta)
$$

tends to zero as $n \rightarrow \infty$ by the boundedness of $|d F(\theta)|$. Thus the lemma is established.

2. In this paragraph, we consider the divergence of interpolation polynomials of a function which belongs to $K_{p}$.

Theorem 1. Let $f(z)$ be a function which belongs to the class $K_{\mathrm{p}}(\rho>1)$ and $(P)$ be the points set which satisfies the condition $(C)$. Then the sequence of polynomials $P_{n}(z ; f)$ of respectively degrees $n$ found by interpolation to $f(z)$ in all the zeros of $w_{n+1}(z)$ diverges at every point exterior to $C_{\mathrm{p}}$. Moreover, we have

$$
\varlimsup_{n \rightarrow \infty}\left|\left(\frac{\rho}{z}\right)^{n} P_{n}(z ; f)\right|>0 \text { for }|z|>\rho>1 .
$$

In the proof of this theorem, it is convenient to have the

Lemma 2.1. Let $f(z)$ be the function of $K_{p}(\rho>1), \lambda(z)$ be a function single valued and analytic exterior to the unit circle $C:|z|=1$ with positive modulus. Let $S_{n}(z ; f)$ be the sequence of functions defined by

Then

$$
S_{n}(z ; f)=\frac{p f .}{2 \pi i} \int_{c_{\rho}} \frac{\lambda(t) t^{n+1}-\lambda(z) z^{n+1}}{\lambda(t) t^{n+1}} \frac{f(t)}{t-z} d t
$$

If $\lambda(z) \equiv 1, S_{n}(z ; f)$ are partial sums of the power series of $f(z)$.

Now we shall prove the lemma. In (2.2), for a fixed point $z$ exterior to $C_{\rho}$,

$$
\left(\frac{\rho}{z}\right)^{n+1} \frac{p f .}{2 \pi i} \int_{p} \frac{f(t)}{t-z} d t
$$

converges clearly to zero as $n \rightarrow \infty$. And

$$
\left(\frac{\rho}{z}\right)^{n+1} \frac{p f \cdot \int_{0}}{2 \pi i} \int_{c_{\rho}} \frac{\lambda(z) z^{n+1}}{\lambda(t) t^{n+1}} \frac{f(t)}{t-z} d t=\rho^{n+1} \lambda(z) \frac{p f .}{2 \pi i} \int_{c_{\rho}}[\lambda(t)(t-z)]^{-1} f(t) t^{-n-1} d t
$$

does not tend to zero as $n \rightarrow \infty$ by lemma 1.2. Now the relation (2.3) follows at once. Thus the lemma is established. 
Now we are in a position to prove the theorem. The sequence of polynomials $P_{n}(z ; f)$ of respective degrees $n$ which interpolate to $f(z)$ in all the zeros of $w_{n+1}(z)$ is given by

$$
P_{n}(z ; f)=\frac{p f \cdot}{2 \pi i} \int_{c_{\rho}} \frac{w_{n+1}(t)-w_{n+1}(z)}{w_{n+1}(t)} \frac{f(t)}{t-z} d t
$$

Then we have

$$
S_{n}(z ; f)-P_{n}(z ; f)=\frac{p f .}{2 \pi i} \int_{C_{p}}\left\{\frac{w_{n+1}(z)}{w_{n+1}(t)}-\frac{\lambda(z) z^{n+1}}{\lambda(t) t^{n+1}}\right\} \frac{f(t)}{t-z} d t
$$

for $z$ exterior to the unit circle $C$. And we have

$$
\begin{aligned}
\left(\frac{\rho}{z}\right)^{n+1}\left\{\frac{w_{n+1}(z)}{w_{n+1}(t)}-\frac{\lambda(z) z^{n+1}}{\lambda(t) t^{n+1}}\right\}=\left(\frac{\rho}{t}\right)^{n+1} & \left\{\frac{w_{n+1}(z)}{w_{n+1}(t)} \frac{t^{n+1}}{z^{n+1}}-\frac{\lambda(z)}{\lambda(t)}\right\} \\
=\left(\frac{\rho}{t}\right)^{n+1}\left\{\frac{w_{n+1}(z)}{z^{n+1}}\left(\frac{t^{n+1}}{w_{n+1}(t)}-\frac{1}{\lambda(t)}\right)\right. & \left.+\frac{1}{\lambda(t)}\left(\frac{w_{n+1}(z)}{z^{n+1}}-\lambda(z)\right)\right\} \\
& \equiv\left(\frac{\rho}{t}\right)^{n+1} \varphi_{n}(t, z) ; \quad n=1,2, \ldots,
\end{aligned}
$$

where $\varphi_{n}(t, z)$ is the sequence of functions of $t$, for any fixed $z$ exterior to $C_{\rho}$, single valued and analytic on $C_{\mathrm{p}}$ and tends to zero as $n \rightarrow \infty$ by the condition

$$
\lim _{n \rightarrow \infty} \frac{w_{n}(z)}{z^{n}}=\lambda(z) \neq 0
$$

uniformly for any finite closed points set exterior to $C ;|z|=1$.

Now the relation

(2.6) $\lim _{n \rightarrow \infty}\left(\frac{\rho}{z}\right)^{n}\left\{S_{n}(z ; f)-P_{n}(z ; f)\right\}=\lim _{n \rightarrow \infty} \frac{\rho^{n} p f \cdot}{2 \pi i} \int_{c_{\rho}} \varphi_{n}(t, z) f(t) t^{-n-1} d t=0$

follows at once by lemma 1.3. Now we can verify from (2.3) and (2.6)

$$
\overline{\lim }_{n \rightarrow \infty}\left|\left(\frac{\rho}{z}\right)^{n} P_{n}(z ; f)\right|>0
$$

for $z$ exterior to $C_{\rho}$. That is, the sequence of polynomials $P_{n}(z ; f)$ diverges with the order $\left|\frac{z}{\rho}\right|^{n}$ as $S_{n}(z ; f)$ diverges. Thus the theorem has been established. 